\title{
INFLUENCE OF OVALBUMIN GENE REGULATORY ELEMENTS ON TISSUE SPECIFICITY AND LEVEL OF TRANSGENE EXPRESSION
}

\section{N.A. VOLKOVA, I.K. FOMIN, A.V. DOTSEV, T.E. DENISKOVA, N.A. ZINOVIEVA}

L.K. Ernst All-Russian Research Institute of Animal Husbandry, Federal Agency of Scientific Organizations, 60, pos. Dubrovitsy, Podolsk District, Moscow Province, 142132 Russia, e-mail natavolkova@inbox.ru Acknowledgements:

Supported by Federal Agency of Scientific Organizations

Received September 14, 2016

\section{Abstract}

The use of lentiviral vectors for the genetic modification of embryonic chicken cells is regarded as one of the promising methods for producing transgenic poultry. In this case, it is very important to determine the regulatory elements of the ovalbumin gene, providing tissue-specificity and high transgene expression in cells of chicken oviduct. The aim of this work was to study the effect of the intron sequences and promoter of ovalbumin gene on tissue specificity and level of the transgene expression. For this purpose constructs based on lentiviral vector pWpxl, containing eGFP marker gene under control of the modified ovalbumin gene regulatory elements were obtained. Vector $\mathrm{pW} 2.8$ included a chromosomal DNA fragment of $2.8 \mathrm{~kb}$ comprising a first exon, intron sequence and part of the second exon of the ovalbumin gene; vector pW1.2 - chromosomal DNA fragment of $1.2 \mathrm{~kb}$ comprising a promoter and ovalbumin gene sequence (without the intron and the first exon) to the transcription initiation point; vectors pW131, pW225, pW315 - chromosomal DNA fragment, similar to the fragment of $1.2 \mathrm{~kb}$ in pW1.2 vector in which promoter ( $80 \mathrm{bp})$ was replaced by highly structured sequence of the birds $\beta$-actin gene promoter region of 131,225 or 315 bp respectively. The control vector pWCAGgfp included constitutive hybrid regulatory element comprising human cytomegalovirus early gene enhancer and birds' $\beta$-actin gene promoter (CAG). Primary culture cells of chick oviduct and human fibrosarcoma cells 293T (control) were used as target cells for transfection. Viral preparation was added after a monolayer of cells reached concentration of 1$3 \times 10^{7} \mathrm{CFU} / \mathrm{ml}$. eGFP expression was determined by fluorimetry in 72 hours after transfection. Low level of expression of eGFP gene controlled by chromosomal fragment of $2.8 \mathrm{~kb}$ leader region of the ovalbumin gene was confirmed in vitro using culture of chicken oviduct cells and 293T cell line: in vector pW2.8 recombinant protein expression level was up to 25 times lower compared to pWCAGgfp vector with a constitutive promoter CAG. Yet the eGFP expression levels for pW1.2 and pW2.8 constructs were identical, indicating the absence of introns' influence on the expression level of the recombinant DNA using this regulatory element. When the ovalbumin gene promoter was replaced by highly structured elements of $\beta$-actin constitutive gene promoter the increase in the expression of eGFP in 2-3 times was observed, as well as the increased expression occurred with lengthening of the promoter region of $\beta$-actin gene (vectors pW131, pW225, pW315). The achieved levels of expression with the use of exogenous $\beta$-actin gene promoter were comparable with expression levels controlled by a constitutive promoter CAG, however when the promoter part of the ovalbumin gene was replaced by exogenous promoter (gene $\beta$-actin), the deregulation of tissue-specific expression of eGFP was observed, indicating that transcription with tissue-specific ovalbumin promoter gene can be modulated or activated with exogenous enhancers. ry elements

Keywords: transgenesis, chickens, lentiviral vectors, oviduct, ovalbumin gene regulato-

The use of viral vectors derived from recombinant retroviruses and lentiviruses for genetic modification of poultry is regarded as one of the promising methods for producing transgenic animals. The high efficacy of these vector systems for poultry transgenesis has been shown [1-6]. One of the main directions in the transgenesis of poultry is the development of effective vector systems, which provide the high expression of recombinant protein in the oviduct cells to produce a transgenic product.

A significant expression of recombinant protein in the oviduct cells (up 
to several mg per ml) in transgenic animals $\left(\mathrm{G}_{0}\right)$ was achieved using gene constructs comprising constitutive promoters/enhancers, in particular the CMV (human cytomegalovirus) immediate-early gene enhancer/promoter and chicken $\beta$-actin gene promoter [7]. However decreased expression of recombinant proteins in oviduct cells in subsequent generations $\left(G_{1}\right.$ and $\left.G_{2}\right)$ was observed. A direct correlation between the expression of the recombinant product and a "gene dose" (the number of virus copies per cell genome) has been established, i.e. the intensive production of the transgenic product in organs and tissues of genetically modified animals increases the risk of physiological defects [8-10].

A solution to the problem may be the use of promoters providing tissuespecific expression of recombinant genes as a part of gene constructs. As a promising approach is considered the use of regulatory elements that control egg ovalbumin synthesis, in particular the 7.5 and $2.8 \mathrm{kbp}$ chromosomal DNA fragments flanking the 5'-end of the ovalbumin gene [11-15]. A stable tissue-specific expression of the recombinant gene in several generations of transgenic birds was observed when these DNA fragments were included in the gene constructs. However, the synthesis of the recombinant product was 20-50 times lower compared to the results obtained when using constitutive promoters [16]. It indicates the effect on the ovalbumin synthesis regulation system from several regulatory elements located both inside and next to or outside the structural gene.

We first attempted to investigate the extent of tissue-specific gene expression using various modifications of the $2.8 \mathrm{kbp}$ chromosomal DNA fragment that included about $1.2 \mathrm{kbp}$ located upstream of the initiation point of transcription and $1.6 \mathrm{kbp}$, including the first two exons and an intron of the ovalbumin gene.

The aim of the present work was to study the effect of the intron sequences and promoter of ovalbumin gene contained in the gene constructs on the tissue specificity and expression of recombinant DNA.

Technique. We used a modified lentiviral vector system of the second generation, which included three plasmids, such as psPAX2 (encodes gag and pol genes, is a classic packer), pLPG (encodes a surface glycoprotein G of vesicular stomatitis virus VVS-G) and pWPXL (contains eGFP gene, the enhanced green fluorescence protein), under the control of a promoter of human RNA polymerase II elongation factor-1 $h E F 1 \alpha$, serves as a self-inactivating lentiviral vector) [17]. The pWCAGgfp, pW2.8, pW1.2, pW131, pW225 and pW315 vectors were constructed based on the pWPXL plasmid, lentiviral vectors are standard molecular cloning techniques [18].

To obtain a recombinant virus and determine viral titers, the cell line 293T was used. Cells were cultured in the DMEM medium (Dlbecco's Modified Eagle's Medium), containing fetal calf serum (10\%), L-glutamine (2 mM), penicillin $(100 \mathrm{U} / \mathrm{ml})$, streptomycin $(100 \mu \mathrm{g} / \mathrm{ml})$ in an atmosphere of $5 \% \mathrm{CO}_{2}$ at a temperature of $37^{\circ} \mathrm{C}$. Viral preparations were concentrated, subjecting the culture supernatants to ultracentrifugation $\left(70,000 \mathrm{~g}, 120 \mathrm{~min},+4{ }^{\circ} \mathrm{C}\right)$, pellets were resuspended in the TNE buffer $(50 \mathrm{mM}$ Tris- $\mathrm{HCl}, \mathrm{pH} 7.8 ; 130 \mathrm{mM} \mathrm{NaCl} ; 1 \mathrm{mM}$ $\mathrm{Na}_{2}$-EDTA). The physical titers of viral vectors were determined by a quantitative real-time PCR using the MiniOpticon ${ }^{\mathrm{TM}}$ analyzer (Bio-Rad, USA), and the biological titre of the virus - according to G. Tiscornia et al. [19]. These viral preparations used for primary culture of chicken oviduct cells infected according to the procedure [20].

The primary culture of chick oviduct cells and human fibrosarcoma cells 293T (control) were used as target cells for transfection. Viral preparation (1-3 $\mathrm{CFU} / \mathrm{ml}$ ) was added after cells reached a monolayer.

eGFP fluorescence was measured using the FACSCanto flow cytometer 
(BD, USA), with a set of filters $\lambda=480-490 \mathrm{~nm}$ (excitation) and $\lambda=510 \mathrm{~nm}$ (emission), 72 hours after transfection. To account the expression of $e G F P$, the mean fluorescence intensity (MFI) of cells with expression constructs was determined compared to the original cells.

Results. Based on the pWpxl lentiviral vector, five gene expression constructs were derived containing the eGFP marker gene under control of the modified ovalbumin gene regulatory elements (Table 1).

\section{A characterization of gene constructs derived from lentiviral vector pWpxl ( $e G F P$} used as a structural gene)

\begin{tabular}{|c|c|}
\hline Vector & Regulatory elements \\
\hline pW2.8 & $2.8 \mathrm{kbp}$, includes the first exon, first intron and a part of the second exon of the ovalbumin gene \\
\hline pW1.2 & $\begin{array}{l}1.2 \mathrm{kbp} \text {, includes a promoter and a sequence (without the intron and the first exon) of the ovalbumin } \\
\text { gene upstream to the initiation point of transcription }\end{array}$ \\
\hline pW131 & $\begin{array}{l}1.2 \mathrm{kbp} \text {, similarly to pW1.2, the promoter part ( } 80 \mathrm{bp} \text { ) was replaced by } 131 \mathrm{bps} \text { of a highly structured se- } \\
\text { quence of the promoter region of the chicken } \beta \text {-actin gene }\end{array}$ \\
\hline pW225 & $\begin{array}{l}1.3 \mathrm{kbp} \text {, similarly to pW1.2, the promoter part ( } 80 \mathrm{bps}) \text { was replaced by } 225 \mathrm{bps} \text { of a highly structured se- } \\
\text { quence of the promoter region of the chicken } \beta \text {-actin gene }\end{array}$ \\
\hline pW315 & $\begin{array}{l}1.4 \mathrm{kbp} \text {, similarly to pW1.2, the promoter part ( } 80 \mathrm{bps} \text { ) was replaced by } 315 \mathrm{bps} \text { of a highly structured se- } \\
\text { quence of the promoter region of the chicken } \beta \text {-actin gene }\end{array}$ \\
\hline
\end{tabular}

The control was the pWCAGgfp vector we obtained previously (Figure), which contained the $e G F P$ gene and a hybrid constitutive regulatory element comprising the CMV (human cytomegalovirus) immediate-early gene enhancer and chicken $\beta$-actin gene promoter [21].

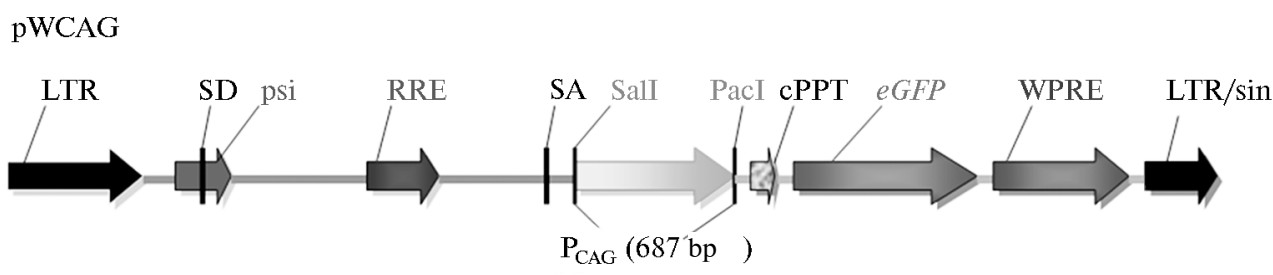

The structure of pWCAG lentiviral expression vector. LTR, LTR/sin (long terminal repeat; sin, self-inactivating): $5^{\prime}$-LTR - wild type, $3^{\prime}$-LTR/sin - self-inactivating variant. SD, SA - splice donor and splice acceptor sites; psi - a region responsible for virion packaging of the viral RNA; RRE - Rev-responsible element, a binding site of the Rev-protein, carrying out transport of the genomic RNA molecule from the nucleus to the cytoplasm; cPPT - central polypurine tract participating in the transport of the pre-integration complex to the cell nucleus; $e G F P$ - enhanced green fluorescence protein; WPRE - Woodchuck hepatitis virus post-transcriptional regulatory element. $\mathrm{P}_{\mathrm{CAG}}$ - the hybrid human cytomegalovirus immediate-early gene enhancer/chicken $\beta$-actin gene promoter. SalI, PacI - restriction sites that were used to clone regulatory elements.

Plasmid DNA of viral vectors, as well as of the packer and VVS-G was isolated in the preparative amounts, then fine purified and assayed to determine the optimal ratio between the components during transfection. When producing viral preparations, a three-plasmid vector system was injected transiently into human cells (293T line) via $\mathrm{CaPO}_{4}$-precipitation. For each of the expression constructs, we derived preparations with high viral titers $\left(1-3 \times 10^{7} \mathrm{CFU} / \mathrm{ml}\right)$ for their further introduction into target cells.

The mean fluorescence intensity in the chicken oviduct cell culture using pWCAGgfp vector with a constitutive promoter/enhancer CAG was 89 times higher than the background intensity in the control (Table 2). When vectors pW2.8 and pW1.2 were introduced into the target cells along with ovalbumin gene regulatory elements, the $e G F P$ expression exceeded the control values by 3.5 times, and was 25 times lower than in the case of a constitutive promoter. The inclusion of highly structured elements of the constitutive promoter $\beta$-actin gene (pW131, pW225 and pW313 vectors) in the ovalbumin gene regulatory elements contributed to a 7.8-14.8-fold increase of expression of the recombinant gene. 
2. Expression of the $\boldsymbol{e G F P}$ marker gene (in mean fluorescence intensity, $U$ ) in the primary chicken oviduct cells and heterologous $293 \mathrm{~T}$ cells when using different vectors with modified ovalbumin gene regulatory elements

\begin{tabular}{l|c|c|c|c|c|c|c}
\hline \multicolumn{1}{c}{ Cell culture } & Control & pWCAGgfp & pW2.8 & pW1.2 & pW131 & pW225 & pW315 \\
\hline $\begin{array}{l}\text { Primary culture of } \\
\text { chicken oviduct cells }\end{array}$ & 128 & 11433 & 459 & 461 & 3601 & 4841 & 6785 \\
293T cell line & 542 & 10936 & 497 & 538 & 1324 & 1508 & 1810 \\
\hline Not e. Control - non-transformed cell culture, background fluorescence. & & & \\
\hline
\end{tabular}

To assess the tissue specificity of the obtained vectors and identify the regulatory elements in the leader region of the ovalbumin gene that are responsible for tissue-specific expression, the vectors prepared were introduced into heterologous 293T cells (human fibrosarcoma cells) by infection. Expression levels of the $e G F P$ marker gene were determined for different expression cassettes. The expression of the marker gene in transfected cells using the pWCAGgfp vector was virtually identical to that obtained in chicken oviduct cells. However, given the relatively high background fluorescence, only a 20-fold excess of control values was found in the test samples. When pW2.8 and pW1.2 vectors, carrying the chromosomal fragments of ovalbumin gene, were introduced, mean fluorescence intensity in the test samples did not differ from the background fluorescence, indicating the lack of expression of the $e G F P$ gene as a result of tissue specificity of the constructs. A 2.5-4.0-fold increase of mean fluorescence in the test samples was observed in the case of pW131, pW225 and pW315 vectors containing fragments of the promoter sequence of the $\beta$-actin gene in the composition of the regulatory elements of the ovalbumin gene. And the portion of fluorescent cells in this case amounted up to $35 \%$ (for pW2.8 and pW1.2 it was 0.65-1.70\%), which indicates a lack of tissue specificity in the used vectors.

Therefore, the results confirmed the low expression of recombinant genes when using regulatory elements of the $2.8 \mathrm{~kb}$ chromosomal DNA fragment from the leader region of the ovalbumin gene. This value was about 25 times lower than for a vector with a constitutive promoter CAG. The level of the eGFP expression in the pW2.8 and pW1.2 constructs was identical, suggesting no influence of the intron on the expression if this regulatory element concerned. The substitution of the ovalbumin gene promoter with highly structured elements of the constitutive promoter of the $\beta$-actin gene contributed to a significant increase in the expression of the recombinant gene. The effect of the expression gain was identified when the promoter region of the $\beta$-actin gene extended. However, the substitution of the promoter part of the ovalbumin gene with an exogenous promoter (the $\beta$-actin gene promoter) resulted in the deregulation of the tissue-specific expression of $e G F P$.

\section{REFERENCES}

1. Mizuarai S., Ono K., Yamaguchi K., Nishiji ma K-I., Kamihira M., I iji $\mathrm{ma}$ S. Production of transgenic quails with high frequency of germ-line transmission using VSV-G pseudotyped retroviral vector. Biochem. Biophys. Res. Commun., 2001, 286: 456-463 (doi: 10.1006/bbrc.2001.5422).

2. Kwon M.S., Koo B.C., Choi B.R., Park Y.Y., Lee Y.M., Suh H.S., Park Y.S., Lee H.T., Kim J.H., Roh J.Y., Kim N.H., Kim T. Generation of transgenic chickens that produce bioactive human granulocyte-colony stimulating factor. Mol. Reprod. Dev., 2008, 75(7): 1120-1126 (doi: 10.1002/mrd.20860).

3. Kwon S.C., Choi J.W., Jang H.J., Shin S.S., Lee S.K., Park T.S., Choi I.Y., Lee G.S., Song G., Han J.Y. Production of biofunctional recombinant human interleukin 1 receptor antagonist (rhIL1RN) from transgenic quail egg white. Biol. Reprod., 2010, 82: 1057-1064 (doi: 10.1095/biolreprod.109.081687).

4. Chapman S.C., Laws on A., Macarthur W.C., Wiese R.J., Loechel R.H., Burgos-Trinidad M., Wakefield J.K., Ramabhadran R., Mauch T.J., 
$\mathrm{S}$ c h o e nw olf G.C. Ubiquitous GFP expression in transgenic chickens using a lentiviral vector. Development, 2005, 132: 935-940 (doi: 10.1242/dev.01652).

5. S m i t h C.A., Ro e s z le r K.N., S i n c la i r A.H. Robust and ubiquitous GFP expression in a single generation of chicken embryos using the avian retroviral vector, RCASBP. Differentiation, 2009, 77(5): 473-482 (doi: 10.1016/j.diff.2009.02.001).

6. Rapp J.C., Harvey A.J., Speksnijder G.L., Hu W., Ivarie R. Biologically active human interferon $\alpha-2 b$ produced in the egg white of transgenic hens. Transgenic Res., 2003, 12(5): 569-575 (doi: 10.1023/A:1025854217349).

7. Kamihira M., Ono K., Esaka K., Nishijima K., Kigaku R., Komatsu H., Yamashita T., Kyogoku K., Iijima S. High-level expression of single-chain Fv-Fc fusion protein in serum and egg white of genetically manipulated chickens by using a retroviral vector. J. Virol., 2005, 79(17): 10864-10874 (doi: 10.1128/JVI.79.17.10864-10874.2005).

8. McGrew M.J., Sherman A., Ellard F.M., Lillico S.G., Gilhooley H.J., Kingsman A.J., M it ro phanous K.A., S a ng H. Efficient production of germline transgenic chickens using lentiviral vectors. EMBO Rep., 2004, 5: 728-733 (doi: 10.1038/sj.embor.7400171).

9. Scott B.B., Velho T.A., Sim S., Lois C. Applications of avian transgenesis. ILAR J., 2010, 51(4): 353-361 (doi: 10.1093/ilar.51.4.353).

10. Furlan-Magaril M., Rebollar E., Guerrero G., Fernandez A., Moltai E., Gonzalez-Buenda E., Cantero M., Montoliu L., Recillas-Targa F. An insulator embedded in the chicken $\alpha$-globin locus regulates chromatin domain configuration and differential gene expression. Nucl. Acids Res., 2011, 39(1): 89-103 (doi: 10.1093/nar/gkq740).

11. Scott B.B., Lois C. Generation of tissue-specific transgenic birds with lentiviral vectors. PNAS, 2005, 102(45): 16443-16447 (doi: 10.1073/pnas.0508437102).

12. Dougherty D.C., S a nders M.M. Estrogen action: revitalization of the chick oviduct model. Trends Endocrinol. Metab., 2005, 16: 414-419 (doi: 10.1016/j.tem.2005.09.001).

13. Shimizu M., Losos J.K., Gibbins A.M. Analysis of an approach to oviduct-specific expression of modified chicken lysozyme genes. Biochem. Cell Biol., 2005, 83(1): 49-60 (doi: 10.1139/o04-122).

14. Byun S.J., Kim S.W., Kim K.W., Kim J.S., Hwang I.S., Chung H.K., Kan I.S., Jeon I.S., Chang W.K., Park S.B., Yoo J.G. Oviduct-specific enhanced green fluorescent protein expression in transgenic chickens. Biosci. Biotechnol. Biochem., 2011, 75(4): 646-649 (doi: 10.1271/bbb.100721).

15. Lillico S.G., Sherman A., McGrew M.J., Robertson C.D., Smith J., Has1a $\mathrm{m}$ C., B a rna rd P., R a d $\mathrm{l}$ iffe P.A., M it rophanous K.A., Elliot E.A., S ang H.M. Oviduct-specific expression of two therapeutic proteins in transgenic hens. PNAS, 2007, 104(6): 1771-1776 (doi: 10.1073/pnas.0610401104).

16. Kodama D., Nishimiya D., Nishijima K., Okino Y., Inayoshi Y., Kojima Y., Ono K., Motono M., Miyake K., Kawabe Y., KyogokuK., Yamashit a T., K a mihira M., I iji m a S. Chicken oviduct-specific expression of transgene by a hybrid ovalbumin enhancer and the Tet expression system. J. Biosci. Bioeng., 2012, 113(2): 146-153 (doi: 10.1016/j.jbiosc.2011.10.006).

17. Schomber T., Kalberer C.P., Wodnar-Filipowicz A., Skoda R.C. Gene silencing by lentivirus-mediated delivery of siRNA in human CD34+ cells. Blood, 2004, 103(12): 45114513 (doi: 10.1182/blood-2003-07-2397).

18. S a m b ro o k J., Fritsch E.F., Maniatis T. Molecular cloning: a laboratory manual. $2^{\text {nd }}$ ed. Cold Spring Harbor, NY, 1989.

19. T i s c o r n i a G., S i n g e r O., V e r m a I.M. Production and purification of lentiviral vectors. Nature Protocols, 2006, 1(1): 241-245 (doi: 10.1038/nprot.2006.37).

20. DNA cloning. Volume III. A practical approach. D.M. Glover (ed.). Oxford, Washington DC, IRL Press Ltd., 1987.

21. Volk o va N.A., Fo m i n I.K., T o m g o rova E.K., V e to kh A.N., M e n n ib a v a E.R., B r e m G., Z i n o vi e v a N.A. The study of factors affected the gene transfer efficiency in chicken embryonic cells by application of lentiviral vectors. Agricultural Biology, 2015, 50(4): 458-466 (doi: 10.15389/agrobiology.2015.4.458eng) (in Engl.). 